\title{
Coefficient estimates and subordination properties for certain classes of analytic functions of reciprocal order
}

\author{
Tariq Al-Hawary and Basem Aref Frasin
}

\begin{abstract}
In this work, we determine the coefficient bounds and subordination results for functions in certain subclasses of analytic functions of reciprocal order, which are introduced here by means of a Hadamard product of analytic functions. The results presented in this paper improve or generalize the recent works of other authors and also give rise to several new results.
\end{abstract}

Mathematics Subject Classification (2010): 30C45, 30C80.

Keywords: Analytic function, starlike function, convex function, $\beta$-spirallike of order $\alpha$, subordinating factor sequence.

\section{Introduction and Preliminaries}

Let $\mathcal{A}$ denote the class of functions $f(z)$ defined by

$$
f(z)=z+\sum_{n=2}^{\infty} a_{n} z^{n}
$$

which are analytic and univalent in the open unit disk $\mathbb{U}=\{z:|z|<1\}$. A function $f \in \mathcal{A}$ is said to be starlike of order $\alpha$ if it satisfies

$$
\operatorname{Re}\left(\frac{z f^{\prime}(z)}{f(z)}\right)>\alpha \quad(0 \leq \alpha<1, z \in \mathbb{U}) .
$$

We denote by $\mathcal{S}^{*}(\alpha)$ the subclass of $\mathcal{A}$ consisting of functions which are starlike of order $\alpha$ in $\mathbb{U}$. Also, a function $f \in \mathcal{A}$ is said to be convex of order $\alpha$ if it satisfies

$$
\operatorname{Re}\left(1+\frac{z f^{\prime \prime}(z)}{f^{\prime}(z)}\right)>\alpha \quad(0 \leq \alpha<1, z \in \mathbb{U}) .
$$


We denote by $\mathcal{K}(\alpha)$ the subclass of $\mathcal{A}$ consisting of functions which are convex of order $\alpha$ in $\mathbb{U}$. Clearly, we have $\mathcal{S}^{*}(\alpha) \subseteq \mathcal{S}^{*}(0)=\mathcal{S}^{*}, \mathcal{K}(\alpha) \subseteq \mathcal{K}(0)=\mathcal{K}$ and $f(z) \in \mathcal{K}(\alpha)$ if and only if $z f^{\prime}(z) \in \mathcal{S}^{*}(\alpha)$ for $0 \leq \alpha<1$.

For $|\beta|<\frac{\pi}{2}$ and $0 \leq \alpha<1$, a function $f \in \mathcal{A}$ is said to be $\beta$-spirallike of order $\alpha$ in $\mathbb{U}$ if it satisfies

$$
\operatorname{Re}\left(e^{i \beta} \frac{z f^{\prime}(z)}{f(z)}\right)>\alpha \cos \beta \quad(z \in \mathbb{U}) .
$$

The class of all such functions is denoted by $\mathcal{S}_{\beta}(\alpha)[8]$.

A function $f \in \mathcal{A}$ is said to be starlike of reciprocal order $\alpha$ if

$$
\operatorname{Re}\left\{\frac{f(z)}{z f^{\prime}(z)}\right\}>\alpha \quad(0 \leq \alpha<1, z \in \mathbb{U}) .
$$

We denote the class of such functions by $\mathcal{S}^{-1}(\alpha)$. Furthermore, a function $f \in \mathcal{A}$ is said to be convex of reciprocal order $\alpha$ if

$$
\operatorname{Re}\left\{\frac{1}{1+\frac{z f^{\prime \prime}(z)}{f^{\prime}(z)}}\right\}>\alpha \quad(0 \leq \alpha<1, z \in \mathbb{U}) .
$$

The class of all such convex functions of reciprocal order $\alpha$ is denoted by $\mathcal{K}^{-1}(\alpha)$.

We note that $f(z) \in \mathcal{K}^{-1}(\alpha)$ if and only if $z f^{\prime}(z) \in \mathcal{S}^{-1} *(\alpha)$.

In view of the fact that

$$
\operatorname{Re} p(z)>0 \Rightarrow \operatorname{Re} \frac{1}{p(z)}=\operatorname{Re} \frac{p(z)}{|p(z)|^{2}}>0
$$

it follows that $\mathcal{S}^{-1} *(0)=\mathcal{S}^{*}$ and $\mathcal{K}^{-1}(0)=\mathcal{K}$. In particular, every starlike function of reciprocal order $\alpha \geq 0$ is starlike and hence univalent.

Example 1.1. The function $f(z)=z e^{(1-\alpha) z}$ is a starlike function of reciprocal order $1 /(2-\alpha)[9$, Example 2].

For functions $f \in \mathcal{A}$ given by (1.1) and $g \in \mathcal{A}$ given by

$$
g(z)=z+\sum_{n=2}^{\infty} b_{n} z^{n}
$$

we define the Hadamard product (or Convolution ) of $f$ and $g$ by

$$
(f * g)(z)=z+\sum_{n=2}^{\infty} a_{n} b_{n} z^{n} .
$$

Motivated and inspired by the work done by Owa et al. [11] and by making use of the Hadamard product (1.7), we now introduce the following subclass of $\mathcal{A}$.

Definition 1.2. Let $\Phi(z)=z+\sum_{n=2}^{\infty} \delta_{n} z^{n}$ and $\Psi(z)=z+\sum_{n=2}^{\infty} \mu_{n} z^{n}$ be analytic in $\mathbb{U}$, such that $\delta_{n} \geq 0, \mu_{n} \geq 0$ and $\delta_{n} \geq \mu_{n}$ for $n \geq 2$, we say that $f(z) \in \mathcal{A}$ is in the class 
$\mathcal{S}^{-1}(\Phi, \Psi ; \alpha, \beta)$ if $f(z) * \Phi(z) \neq 0, \quad f(z) * \Psi(z) \neq 0$ and

$$
\left|\frac{1}{e^{i \beta}\left(\frac{f(z) * \Phi(z)}{f(z) * \Psi(z)}\right)}-\frac{1}{2 \alpha}\right|<\frac{1}{2 \alpha} \quad(\beta \in \mathbb{R}, 0<\alpha<1, z \in \mathbb{U}) .
$$

Several known and new subclasses of analytic functions of reciprocal order $\alpha$ can be obtained from the class $\mathcal{S}^{-1}(\Phi, \Psi ; \alpha, \beta)$, by suitably specializing the values of $\Phi$, $\Psi$ and $\beta$. We present below some of these subclasses of $\mathcal{S}^{-1}(\Phi, \Psi ; \alpha, \beta)$ consisting of functions of the form (1.1).

Example 1.3. If $\Phi(z)=z(1-z)^{-2}$ and $\Psi(z)=z(1-z)^{-1}$, then

$$
\begin{aligned}
& \mathcal{S}^{-1}\left(z(1-z)^{-2}, z(1-z)^{-1} ; \alpha, \beta\right) \\
& \equiv \mathcal{S}_{\beta}(\alpha):=\left\{f \in \mathcal{A}:\left|\frac{e^{-i \beta} f(z)}{z f^{\prime}(z)}-\frac{1}{2 \alpha}\right|<\frac{1}{2 \alpha} \quad(\beta \in \mathbb{R}, 0<\alpha<1, z \in \mathbb{U})\right\} .
\end{aligned}
$$

Example 1.4. If $\Phi(z)=\left(z+z^{2}\right)(1-z)^{-3}$ and $\Psi(z)=z(1-z)^{-2}$, then

$$
\begin{aligned}
& \mathcal{S}^{-1}\left(\left(z+z^{2}\right)(1-z)^{-3}, z(1-z)^{-2} ; \alpha, \beta\right) \\
& \equiv \mathcal{K}_{\beta}(\alpha):=\left\{f \in \mathcal{A}:\left|\frac{e^{-i \beta} f^{\prime}(z)}{f^{\prime}(z)+z f^{\prime \prime}(z)}-\frac{1}{2 \alpha}\right|<\frac{1}{2 \alpha} \quad(\beta \in \mathbb{R}, 0<\alpha<1, z \in \mathbb{U})\right\} .
\end{aligned}
$$

The class $\mathcal{S}_{\beta}(\alpha)$, the $\beta$-spirallike functions of reciprocal order $\alpha$ and the class $\mathcal{K}_{\beta}(\alpha)$, the $\beta$-convexlike functions of reciprocal order $\alpha$ were studied by Owa et al. [11].

Example 1.5. If $\Phi(z)=z(1-z)^{-2}, \Psi(z)=z(1-z)^{-1}$ and $\beta=0$, then

$$
\begin{aligned}
& \mathcal{S}^{-1}\left(z(1-z)^{-2}, z(1-z)^{-1} ; \alpha, 0\right) \\
& \equiv \mathcal{M}(\alpha):=\left\{f \in \mathcal{A}:\left|\frac{f^{\prime}(z)}{z f(z)}-\frac{1}{2 \alpha}\right|<\frac{1}{2 \alpha} \quad(0<\alpha<1, z \in \mathbb{U})\right\} .
\end{aligned}
$$

Example 1.6. If $\Phi(z)=\left(z+z^{2}\right)(1-z)^{-3}, \Psi(z)=z(1-z)^{-2}$ and $\beta=0$, then

$$
\begin{aligned}
& \mathcal{S}^{-1}\left(\left(z+z^{2}\right)(1-z)^{-3}, z(1-z)^{-2} ; \alpha, 0\right) \\
& \equiv \mathcal{N}(\alpha):=\left\{f \in \mathcal{A}:\left|\frac{f^{\prime}(z)}{f^{\prime}(z)+z f^{\prime \prime}(z)}-\frac{1}{2 \alpha}\right|<\frac{1}{2 \alpha} \quad(0<\alpha<1, z \in \mathbb{U})\right\} .
\end{aligned}
$$

The classes $\mathcal{M}(\alpha)$ and $\mathcal{N}(\alpha)$ were studied by Owa et al. [10].

Furthermore, we have the following new classes:

Example 1.7. If $\Phi(z)=z(1-z)^{-2}$ and $\Psi(z)=z$, then

$$
\begin{aligned}
& \mathcal{S}^{-1}\left(z(1-z)^{-2}, z ; \alpha, \beta\right) \\
& \equiv \mathcal{P}_{\beta}(\alpha):=\left\{f \in \mathcal{A}:\left|\frac{1}{e^{i \beta} f^{\prime}(z)}-\frac{1}{2 \alpha}\right|<\frac{1}{2 \alpha} \quad(\beta \in \mathbb{R}, 0<\alpha<1, z \in \mathbb{U})\right\} .
\end{aligned}
$$


Example 1.8. If $\Phi(z)=\left(z+z^{2}\right)(1-z)^{-3}$ and $\Psi(z)=z$, then

$$
\begin{aligned}
& \left.\mathcal{S}^{-1}\left(z+z^{2}\right)(1-z)^{-3}, z ; \alpha, \beta\right) \\
& \equiv \mathcal{R}_{\beta}(\alpha):=\left\{f \in \mathcal{A}:\left|\frac{1}{e^{i \beta}\left(\left(z f^{\prime}(z)\right)^{\prime}\right)}-\frac{1}{2 \alpha}\right|<\frac{1}{2 \alpha} \quad(\beta \in \mathbb{R}, 0<\alpha<1, z \in \mathbb{U})\right\} .
\end{aligned}
$$

In fact many new subclasses of functions of reciprocal order can be defined and studied by suitably choosing $\Phi(z), \Psi(z)$ and $\beta$.

The aim of the present paper is to investigate the coefficient estimates and subordination properties for the class $\mathcal{S}^{-1}(\Phi, \Psi ; \alpha, \beta)$. Some interesting consequences of the results are also pointed out.

\section{Coefficient Estimates}

The sufficient condition for $f(z)$ to be in the class $\mathcal{S}^{-1}(\Phi, \Psi ; \alpha, \beta)$ is given by using coefficient inequalities.

Theorem 2.1. If $f \in \mathcal{A}$ satisfies

$$
\sum_{n=2}^{\infty}\left[\delta_{n}+\left|\delta_{n}-2 \alpha e^{-i \beta} \mu_{n}\right|\right]\left|a_{n}\right| \leq 1-\left|1-2 \alpha e^{-i \beta}\right|
$$

for some $|\beta|<\frac{\pi}{2}$ and $0<\alpha<\cos \beta$, then $f(z) \in \mathcal{S}^{-1}(\Phi, \Psi ; \alpha, \beta)$.

Proof. It suffices to show that

$$
\left|\frac{2 \alpha(f(z) * \Psi(z))-e^{i \beta}(f(z) * \Phi(z))}{e^{i \beta}(f(z) * \Phi(z))}\right|<1 .
$$

We observe that

$$
\begin{aligned}
\left|\frac{2 \alpha(f(z) * \Psi(z))-e^{i \beta}(f(z) * \Phi(z))}{e^{i \beta}(f(z) * \Phi(z))}\right| & =\left|\frac{\left(1-2 \alpha e^{-i \beta}\right)+\sum_{n=2}^{\infty}\left(\delta_{n}-2 \alpha e^{-i \beta} \mu_{n}\right) a_{n} z^{n-1}}{1+\sum_{n=2}^{\infty} \delta_{n} a_{n} z^{n-1}}\right| \\
& \leq \frac{\left|1-2 \alpha e^{i \beta}\right|+\sum_{n=2}^{\infty}\left|\delta_{n}-2 \alpha e^{-i \beta} \mu_{n}\right|\left|a_{n}\right||z|^{n-1}}{1-\sum_{n=2}^{\infty} \delta_{n}\left|a_{n}\right||z|^{n-1}} \\
& <\frac{\left|1-2 \alpha e^{i \beta}\right|+\sum_{n=2}^{\infty}\left|\delta_{n}-2 \alpha e^{-i \beta} \mu_{n}\right|\left|a_{n}\right|}{1-\sum_{n=2}^{\infty} \delta_{n}\left|a_{n}\right|}
\end{aligned}
$$

It follows that the last term is bounded by 1 if

$$
\sum_{n=2}^{\infty}\left[\delta_{n}+\left|\delta_{n}-2 \alpha e^{-i \beta} \mu_{n}\right|\right]\left|a_{n}\right| \leq 1-\left|1-2 \alpha e^{-i \beta}\right|
$$


for some $|\beta|<\frac{\pi}{2}$ and $0<\alpha<\cos \beta$, which is equivalent to (2.1). Therefore, we have $f(z) \in \mathcal{S}^{-1}(\Phi, \Psi ; \alpha, \beta)$ for some $|\beta|<\frac{\pi}{2}$ and $0<\alpha<\cos \beta$.

In the view of Examples 1.3 to 1.8 , we state the following corollaries.

Corollary 2.2. ([11]) If $f \in \mathcal{A}$ satisfies

$$
\sum_{n=2}^{\infty}\left[n+\left|n-2 \alpha e^{-i \beta}\right|\right]\left|a_{n}\right| \leq 1-\left|1-2 \alpha e^{-i \beta}\right|
$$

for some $|\beta|<\frac{\pi}{2}$ and $0<\alpha<\cos \beta$, then $f(z) \in \mathcal{S}_{\beta}(\alpha)$.

Corollary 2.3. ([11]) If $f \in \mathcal{A}$ satisfies

$$
\sum_{n=2}^{\infty} n\left[n+\left|n-2 \alpha e^{-i \beta}\right|\right]\left|a_{n}\right| \leq 1-\left|1-2 \alpha e^{-i \beta}\right|
$$

for some $|\beta|<\frac{\pi}{2}$ and $0<\alpha<\cos \beta$, then $f(z) \in \mathcal{K}_{\beta}(\alpha)$.

Corollary 2.4. ([10]) Let $0<\alpha<1$. If $f \in \mathcal{A}$ satisfies

$$
\sum_{n=2}^{\infty}(n-\alpha)\left|a_{n}\right| \leq \frac{1}{2}(1-|1-2 \alpha|)=\left\{\begin{array}{c}
\alpha ; \quad \text { if } \quad 0<\alpha \leq \frac{1}{2} \\
1-\alpha ; \quad \text { if } \quad \frac{1}{2} \leq \alpha<1
\end{array},\right.
$$

then $f(z) \in \mathcal{M}(\alpha)$.

Corollary 2.5. ([10]) Let $0<\alpha<1$. If $f \in \mathcal{A}$ satisfies

$$
\sum_{n=2}^{\infty} n(n-\alpha)\left|a_{n}\right| \leq \frac{1}{2}(1-|1-2 \alpha|)=\left\{\begin{array}{c}
\alpha ; \quad \text { if } \quad 0<\alpha \leq \frac{1}{2} \\
1-\alpha ; \quad \text { if } \quad \frac{1}{2} \leq \alpha<1
\end{array}\right.
$$

then $f(z) \in \mathcal{N}(\alpha)$.

Corollary 2.6. If $f \in \mathcal{A}$ satisfies

$$
\sum_{n=2}^{\infty} 2 n\left|a_{n}\right| \leq 1-\left|1-2 \alpha e^{-i \beta}\right|
$$

for some $|\beta|<\frac{\pi}{2}$ and $0<\alpha<\cos \beta$, then $f(z) \in \mathcal{P}_{\beta}(\alpha)$.

Corollary 2.7. If $f \in \mathcal{A}$ satisfies

$$
\sum_{n=2}^{\infty} 2 n^{2}\left|a_{n}\right| \leq 1-\left|1-2 \alpha e^{-i \beta}\right|
$$

for some $|\beta|<\frac{\pi}{2}$ and $0<\alpha<\cos \beta$, then $f(z) \in \mathcal{R}_{\beta}(\alpha)$. 


\section{Subordination Results}

To proceed our main result in this section, let us first recall the following definitions and lemma.

Definition 3.1. (Subordination Principle). For two functions $f$ and $g$, analytic in $\mathbb{U}$, we say that the function $f(z)$ is subordinate to $g(z)$ in $\mathbb{U}$, and write $f \prec g$ or $f(z) \prec g(z)$ $(z \in \mathbb{U})$, if there exists a Schwarz function $w(z)$, analytic in $\mathbb{U}$ with $w(0)=0$ and $|w|<1(z \in \mathbb{U})$, such that $f(z)=g(w(z))(z \in \mathbb{U})$. In particular, if the function $g$ is univalent in $\mathbb{U}$, the above subordination is equivalent to $f(0)=g(0)$ and $f(\mathbb{U}) \subset g(\mathbb{U})$.

Definition 3.2. A sequence $\left\{b_{n}\right\}_{n=1}^{\infty}$ of complex numbers is said to be a subordinating factor sequence if, whenever $f(z)$ of the form (1.1), $a_{1}=1$ is analytic, univalent and convex in $\mathbb{U}$, we have the subordination given by

$$
\sum_{n=1}^{\infty} b_{n} a_{n} z^{n} \prec f(z), \quad z \in \mathbb{U}
$$

Lemma 3.3. ([14]) The sequence $\left\{b_{n}\right\}_{n=1}^{\infty}$ is a subordinating factor sequence if and only if

$$
\operatorname{Re}\left\{1+2 \sum_{n=1}^{\infty} b_{n} z^{n}\right\}>0 \quad(z \in \mathbb{U})
$$

Let $\mathcal{S}^{*^{-1}}(\Phi, \Psi ; \alpha, \beta) \subseteq \mathcal{S}^{-1}(\Phi, \Psi ; \alpha, \beta)$ denote the subclass of functions $f \in \mathcal{A}$ whose coefficients $a_{n}$ satisfy the inequalities (2.1) .

Employing the techniques used by Attiya [3], Singh [12] and Srivastava and Attiya [13] ( see also, [1], [2], [4], [5], [6] and [7]), we state and prove the following theorem.

Theorem 3.4. Let $f(z) \in \mathcal{S}^{{ }^{-1}}(\Phi, \Psi ; \alpha, \beta)$ and $\delta_{n}+\left|\delta_{n}-2 \alpha e^{-i \beta} \mu_{n}\right|$ is increasing function for $n \geq 2,|\beta|<\frac{\pi}{2}, 0<\alpha<\cos \beta$. Then

$$
\frac{\delta_{2}+\left|\delta_{2}-2 \alpha e^{-i \beta} \mu_{2}\right|}{2\left(1+\delta_{2}-\left|1-2 \alpha e^{-i \beta}\right|+\left|\delta_{2}-2 \alpha e^{-i \beta} \mu_{2}\right|\right)}(f * g)(z) \prec g(z)
$$

for every function $g(z)$ in the class $\mathcal{K}$ and

$$
\operatorname{Ref}(z)>-\frac{1+\delta_{2}-\left|1-2 \alpha e^{-i \beta}\right|+\left|\delta_{2}-2 \alpha e^{-i \beta} \mu_{2}\right|}{\delta_{2}+\left|\delta_{2}-2 \alpha e^{-i \beta} \mu_{2}\right|}
$$

for $z \in \mathbb{U}$.

The constant $\frac{\delta_{2}+\left|\delta_{2}-2 \alpha e^{-i \beta} \mu_{2}\right|}{2\left(1+\delta_{2}-\left|1-2 \alpha e^{-i \beta}\right|+\left|\delta_{2}-2 \alpha e^{-i \beta} \mu_{2}\right|\right)}$ cannot be replace by any larger one. 
Proof. Let $f(z)=z+\sum_{n=2}^{\infty} a_{n} z^{n} \in \mathcal{S}^{*^{-1}}(\Phi, \Psi ; \alpha, \beta)$, and let $g(z)=z+\sum_{n=2}^{\infty} c_{n} z^{n} \in \mathcal{K}$. Then

$$
\begin{aligned}
& \frac{\delta_{2}+\left|\delta_{2}-2 \alpha e^{-i \beta} \mu_{2}\right|}{2\left(1+\delta_{2}-\left|1-2 \alpha e^{-i \beta}\right|+\left|\delta_{2}-2 \alpha e^{-i \beta} \mu_{2}\right|\right)}(f * g)(z) \\
& =\frac{\delta_{2}+\left|\delta_{2}-2 \alpha e^{-i \beta} \mu_{2}\right|}{2\left(1+\delta_{2}-\left|1-2 \alpha e^{-i \beta}\right|+\left|\delta_{2}-2 \alpha e^{-i \beta} \mu_{2}\right|\right)}\left(z+\sum_{n=2}^{\infty} a_{n} c_{n} z^{n}\right) .
\end{aligned}
$$

Thus, by Definition 3.2, the assertion of our theorem will hold if the sequence

$$
\left\{\frac{\delta_{2}+\left|\delta_{2}-2 \alpha e^{-i \beta} \mu_{2}\right|}{2\left(1+\delta_{2}-\left|1-2 \alpha e^{-i \beta}\right|+\left|\delta_{2}-2 \alpha e^{-i \beta} \mu_{2}\right|\right)} a_{n}\right\}_{n=1}^{\infty}
$$

is a subordinating factor sequence, with $a_{1}=1$. In view of Lemma 3.3, this will be the case if and only if

$$
\operatorname{Re}\left\{1+\sum_{n=1}^{\infty} \frac{\delta_{2}+\left|\delta_{2}-2 \alpha e^{-i \beta} \mu_{2}\right|}{1+\delta_{2}-\left|1-2 \alpha e^{-i \beta}\right|+\left|\delta_{2}-2 \alpha e^{-i \beta} \mu_{2}\right|} a_{n} z^{n}\right\}>0 \quad(z \in \mathbb{U}) .
$$

Since $\delta_{n}+\left|\delta_{n}-2 \alpha e^{-i \beta} \mu_{n}\right|$ increasing for all $n \geq 2,|\beta|<\frac{\pi}{2}, 0<\alpha<\cos \beta$, we obtain

$$
\begin{aligned}
& \operatorname{Re}\left\{1+\sum_{n=1}^{\infty} \frac{\delta_{2}+\left|\delta_{2}-2 \alpha e^{-i \beta} \mu_{2}\right|}{1+\delta_{2}-\left|1-2 \alpha e^{-i \beta}\right|+\left|\delta_{2}-2 \alpha e^{-i \beta} \mu_{2}\right|} a_{n} z^{n}\right\} \\
& =\operatorname{Re}\left\{1+\frac{\delta_{2}+\left|\delta_{2}-2 \alpha e^{-i \lambda} \mu_{2}\right|}{1+\delta_{2}-\left|1-2 \alpha e^{-i \lambda}\right|+\left|\delta_{2}-2 \alpha e^{-i \lambda} \mu_{2}\right|} z+\right. \\
& \left.\frac{1}{1+\delta_{2}-\left|1-2 \alpha e^{-i \beta}\right|+\left|\delta_{2}-2 \alpha e^{-i \beta} \mu_{2}\right|} \sum_{n=2}^{\infty}\left(\delta_{2}+\left|\delta_{2}-2 \alpha e^{-i \beta} \mu_{2}\right|\right) a_{n} z^{n}\right\} \\
& \geq 1-\frac{\delta_{2}+\left|\delta_{2}-2 \alpha e^{-i \beta} \mu_{2}\right|}{1+\delta_{2}-\left|1-2 \alpha e^{-i \beta}\right|+\left|\delta_{2}-2 \alpha e^{-i \beta} \mu_{2}\right|} r- \\
& \frac{1}{1+\delta_{2}-\left|1-2 \alpha e^{-i \beta}\right|+\left|\delta_{2}-2 \alpha e^{-i \beta} \mu_{2}\right|} \sum_{n=2}^{\infty}\left(\delta_{2}+\left|\delta_{2}-2 \alpha e^{-i \beta} \mu_{2}\right|\right)\left|a_{n}\right| r^{n} \\
& >1-\frac{\delta_{2}+\left|\delta_{2}-2 \alpha e^{-i \beta} \mu_{2}\right|}{1+\delta_{2}-\left|1-2 \alpha e^{-i \beta}\right|+\left|\delta_{2}-2 \alpha e^{-i \beta} \mu_{2}\right|} r \\
& -\frac{1-\left|1-2 \alpha e^{-i \beta}\right|}{1+\delta_{2}-\left|1-2 \alpha e^{-i \beta}\right|+\left|\delta_{2}-2 \alpha e^{-i \beta} \mu_{2}\right|} r>0, \quad|z|=r<1 .
\end{aligned}
$$

This evidently proves the inequality (3.5) and hence also the subordination result (3.3). The inequality (3.4) follows from (3.3) by taking $g(z)=\frac{z}{1-z}$.

To prove the sharpness of the constant $\frac{\delta_{2}+\left|\delta_{2}-2 \alpha e^{-i \beta} \mu_{2}\right|}{2\left(1+\delta_{2}-\left|1-2 \alpha e^{-i \beta}\right|+\left|\delta_{2}-2 \alpha e^{-i \beta} \mu_{2}\right|\right)}$, we consider the function

$$
f_{0}(z)=z-\frac{1-\left|1-2 \alpha e^{-i \beta}\right|}{\delta_{2}+\left|\delta_{2}-2 \alpha e^{-i \beta} \mu_{2}\right|} z^{2} \quad\left(|\beta|<\frac{\pi}{2}, 0<\alpha<\cos \beta\right),
$$


which is a member of the class $\mathcal{S}^{*-1}(\Phi, \Psi ; \alpha, \beta)$. Thus from the relation (3.3), we obtain

$$
\frac{\delta_{2}+\left|\delta_{2}-2 \alpha e^{-i \beta} \mu_{2}\right|}{2\left(1+\delta_{2}-\left|1-2 \alpha e^{-i \beta}\right|+\left|\delta_{2}-2 \alpha e^{-i \beta} \mu_{2}\right|\right)} f_{0}(z) \prec \frac{z}{1-z} .
$$

It can be verified that

$$
\min _{z \in \mathbb{U}}\left\{\operatorname{Re}\left(\frac{\delta_{2}+\left|\delta_{2}-2 \alpha e^{-i \beta} \mu_{2}\right|}{2\left(1+\delta_{2}-\left|1-2 \alpha e^{-i \beta}\right|+\left|\delta_{2}-2 \alpha e^{-i \beta} \mu_{2}\right|\right)} f_{0}(z)\right)\right\}=\frac{-1}{2} .
$$

This shows that the constant $\frac{\delta_{2}+\left|\delta_{2}-2 \alpha e^{-i \beta} \mu_{2}\right|}{2\left(1+\delta_{2}-\left|1-2 \alpha e^{-i \beta}\right|+\left|\delta_{2}-2 \alpha e^{-i \beta} \mu_{2}\right|\right)}$ is best possible.

By taking different choices of $\Phi, \Psi$ and $\beta$ in Theorem 3.4 and in view of the Examples 1.3 to 1.8 in Section 1, we state the following corollaries for the subclasses defined in those examples.

Corollary 3.5. Let the function $f(z)$ defined by (1.1) be in the class $\mathcal{S}_{\beta}^{-1}(\alpha) ;|\beta|<\frac{\pi}{2}$, $0<\alpha<\cos \beta$, where $\mathcal{S}_{\beta}^{-1}(\alpha)$ denote the subclasses of functions whose coefficients $a_{n}$ satisfy the inequalities (2.2) and suppose that $g(z) \in \mathcal{K}$. Then

$$
\frac{1+\left|1-\alpha e^{-i \beta}\right|}{3-\left|1-2 \alpha e^{-i \beta}\right|+2\left|1-\alpha e^{-i \beta}\right|}(f * g)(z) \prec g(z)
$$

and

$$
\operatorname{Ref}(z)>-\frac{3-\left|1-2 \alpha e^{-i \beta}\right|+2\left|1-\alpha e^{-i \beta}\right|}{2\left(1+\left|1-\alpha e^{-i \beta}\right|\right)} .
$$

The constant factor $\frac{1+\left|1-\alpha e^{-i \beta}\right|}{3-\left|1-2 \alpha e^{-i \beta}\right|+2\left|1-\alpha e^{-i \beta}\right|}$ in the subordination result (3.6) cannot be replaced by a larger one.

Corollary 3.6. Let the function $f(z)$ defined by (1.1) be in the class $\mathcal{K}_{\beta}^{-1}(\alpha) ;|\beta|<\frac{\pi}{2}$, $0<\alpha<\cos \beta$, where $\mathcal{K}_{\beta}^{-1}(\alpha)$ denote the subclasses of functions whose coefficients $a_{n}$ satisfy the inequalities (2.3) and suppose that $g(z) \in \mathcal{K}$. Then

$$
\frac{2\left(1+\left|1-\alpha e^{-i \beta}\right|\right)}{5-\left|1-2 \alpha e^{-i \beta}\right|+4 \mid 1-\alpha e^{-i \beta \mid}}(f * g)(z) \prec g(z)
$$

and

$$
\operatorname{Ref}(z)>-\frac{5-\left|1-2 \alpha e^{-i \beta}\right|+4\left|1-\alpha e^{-i \beta}\right|}{4\left(1+\left|1-\alpha e^{-i \beta}\right|\right)} .
$$

The constant factor $\frac{2\left(1+\left|1-\alpha e^{-i \beta}\right|\right)}{5-\left|1-2 \alpha e^{-i \beta}\right|+4\left|1-\alpha e^{-i \beta}\right|}$ in the subordination result (3.8) cannot be replaced by a larger one.

Corollary 3.7. Let the function $f(z)$ defined by (1.1) be in the class $\mathcal{M}^{-1}(\alpha) ; 0<\alpha<$ 1 , where $\mathcal{M}^{-1}(\alpha)$ denote the subclasses of functions whose coefficients $a_{n}$ satisfy the inequalities (2.4) and suppose that $g(z) \in \mathcal{K}$. Then

$$
\frac{2-\alpha}{5-2 \alpha-|1-2 \alpha|}(f * g)(z) \prec g(z)
$$


and

$$
\operatorname{Ref}(z)>-\frac{5-2 \alpha-|1-2 \alpha|}{2(1-\alpha)} .
$$

The constant factor $\frac{2-\alpha}{5-2 \alpha-|1-2 \alpha|}$ in the subordination result (3.10) cannot be replaced by a larger one.

Corollary 3.8. Let the function $f(z)$ defined by (1.1) be in the class $\mathcal{N}^{-1}(\alpha) ; 0<\alpha<$ 1 , where $\mathcal{N}^{-1}(\alpha)$ denote the subclasses of functions whose coefficients $a_{n}$ satisfy the inequalities (2.5) and suppose that $g(z) \in \mathcal{K}$. Then

$$
\frac{2(2-\alpha)}{9-4 \alpha-|1-2 \alpha|}(f * g)(z) \prec g(z)
$$

and

$$
\operatorname{Ref}(z)>-\frac{9-4 \alpha-|1-2 \alpha|}{4(2-\alpha)} .
$$

The constant factor $\frac{2(2-\alpha)}{9-4 \alpha-|1-2 \alpha|}$ in the subordination result (3.12) cannot be replaced by a larger one.

Corollary 3.9. Let the function $f(z)$ defined by (1.1) be in the class $\mathcal{P}_{\beta}^{-1}(\alpha) ;|\beta|<\frac{\pi}{2}$, $0<\alpha<\cos \beta$, where $\mathcal{P}_{\beta}^{-1}(\alpha)$ denote the subclasses of functions whose coefficients $a_{n}$ satisfy the inequalities (2.6) and suppose that $g(z) \in \mathcal{K}$. Then

$$
\frac{2}{5-\mid 1-2 \alpha e^{-i \beta \mid}}(f * g)(z) \prec g(z)
$$

and

$$
\operatorname{Ref}(z)>-\frac{5-\left|1-2 \alpha e^{-i \beta}\right|}{4} .
$$

The constant factor $\frac{2}{5-\mid 1-2 \alpha e^{-i \beta \mid}}$ in the subordination result (3.14) cannot be replaced by a larger one.

Corollary 3.10. Let the function $f(z)$ defined by (1.1) be in the class $\mathcal{R}_{\beta}^{-1}(\alpha) ;|\beta|<\frac{\pi}{2}$, $0<\alpha<\cos \beta$, where $\mathcal{R}_{\beta}^{-1}(\alpha)$ denote the subclasses of functions whose coefficients $a_{n}$ satisfy the inequalities (2.7) and suppose that $g(z) \in \mathcal{K}$. Then

$$
\frac{4}{9-\mid 1-2 \alpha e^{-i \beta \mid}}(f * g)(z) \prec g(z)
$$

and

$$
\operatorname{Ref}(z)>-\frac{9-\left|1-2 \alpha e^{-i \beta}\right|}{8} .
$$

The constant factor $\frac{4}{9-\mid 1-2 \alpha e^{-i \beta \mid}}$ in the subordination result (3.16) cannot be replaced by a larger one.

Acknowledgements. The authors would like to thank the referee for his helpful comments and suggestions. 


\section{References}

[1] Aouf, M.K., Subordination properties for a certain class of analytic functions defined by the Salagean operator, Applied Mathematics Letters, 22(2009), 1581-1585.

[2] Aouf, M.K., Shamandy, A., Mostafa, A.O., Adwan, E.A., Subordination theorem of analytic functions defined by convolution, Complex Anal. Oper. Theory, 7(2013), 1117-1126.

[3] Attiya, A.A., On some application of a subordination theorems, J. Math. Anal. Appl., 311(2005), 489-494.

[4] Frasin, B.A., A subordination result for a class of analytic functions, Acta Univ. Apulensis Math. Inform., 29(2012), 99-103.

[5] Frasin, B.A., Subordination results for a class of analytic functions defined by a linear operator, J. Inequal. Pure Appl. Math., 7(2006), no. 4, Article 134, 7 pp.

[6] Frasin, B.A., Darus, M., Subordination results on subclasses concerning Sakaguchi functions, J. Inequal. Appl. 2009, Art. ID 574014, 7 pp.

[7] Frasin, B.A., Talafha, Y., Al-Hawary Tariq, Subordination results for classes of functions of reciprocal order, Tamsui Oxford Journal of Information and Mathematical Sciences, 30(2014), 81-89.

[8] Libera, R.J., Univalent spiral functions, Canad. J. Math., 19(1967), 725-733.

[9] Nunokawa, M., Owa, S., Nishiwaki, J., Kuroki, K. Hayami, T., Differential subordination and argumental property, Comput. Math. Appl., 56(10)(2008), 2733-2736.

[10] Owa S., Ochiai, K., Srivastava, H.M., Some coefficient inequalities and distortion bounds associated with certain new subclasses of analytic functions, Mathematical Inequalities \& Applications, $\mathbf{9}(2006), 125-135$.

[11] Owa S., Sağsőz, F., Kamali, M., On some results for subclass of $\beta$-spirallike functions of order $\alpha$, Tamsui Oxford Journal of Information and Mathematical Sciences, 28(2012), no. 1, 79-93.

[12] Singh, S., A subordination theorems for spirallike functions, Int. J. Math. and Math. Sci., 24(7) (2000), 433-435.

[13] Srivastava, H.M., Attiya, A.A., Some subordination results associated with certain subclasses of analytic functions, J. Inequal. Pure Appl. Math., 5(4)(2004), Article 82, 1-6.

[14] Wilf, H.S. Subordinating factor sequences for convex maps of the unit circle, Proc. Amer. Math. Soc., 12(1961), 689-693.

Tariq Al-Hawary

Ajloun College, Al-Balqa Applied University, Department of Applied Science,

26816 Ajloun, Jordan

e-mail: tariq_amh@bau.edu.jo

Basem Aref Frasin

Al al-Bayt University, Faculty of Science, Department of Mathematics

25113 Mafraq, Jordan

e-mail: bafrasin@yahoo.com 